\title{
The Local Theorem for Monotypic Tilings
}

\author{
Nikolai Dolbilin* \\ Steklov Mathematical Institute \\ Gubkin 8 \\ Moscow 117966, Russia \\ dolbilin@mi.ras.ru \\ and \\ Egon Schulte ${ }^{\dagger}$ \\ Northeastern University \\ Department of Mathematics \\ Boston, MA 02115, USA \\ schulte@neu.edu \\ Submitted: Jun 4, 2004; Accepted: 29 Sep, 2004; Published: 7 Oct, 2004 \\ Mathematics Subject Classification: $52 \mathrm{C} 22$
}

With best wishes to Richard Stanley for his 60th birthday.

\begin{abstract}
A locally finite face-to-face tiling $\mathcal{T}$ of euclidean $d$-space $\mathbb{E}^{d}$ is monotypic if each tile of $\mathcal{T}$ is a convex polytope combinatorially equivalent to a given polytope, the combinatorial prototile of $\mathcal{T}$. The paper describes a local characterization of combinatorial tile-transitivity of monotypic tilings in $\mathbb{E}^{d}$; the result is the Local Theorem for Monotypic Tilings. The characterization is expressed in terms of combinatorial symmetry properties of large enough neighborhood complexes of tiles. The theorem sits between the Local Theorem for Tilings, which describes a local characterization of isohedrality (tile-transitivity) of monohedral tilings (with a single isometric prototile) in $\mathbb{E}^{d}$, and the Extension Theorem, which gives a criterion for a finite monohedral complex of polytopes to be extendable to a global isohedral tiling of space.
\end{abstract}

\footnotetext{
*Supported, in part, by RFBR grants 02-01-00803, 03-01-00463 and SSS 2185.2003.1.

${ }^{\dagger}$ Supported, in part, by NSA-grant H98230-04-1-0116
} 


\section{Introduction}

The local characterization of a global property of a spatial structure is usually a challenging problem. In the context of monohedral tilings in euclidean $d$-space $\mathbb{E}^{d}$, certain global symmetry properties can be detected locally. The Local Theorem for Tilings says that a tiling in $\mathbb{E}^{d}$ is isohedral if and only if the large enough neighborhoods of tiles satisfy certain conditions; see Theorem 4.1 for a precise statement, as well as Section 4 for general comments. This result is closely related to the Local Theorem for Delone Sets, which locally characterizes those sets among the uniformly discrete sets in $\mathbb{E}^{d}$ that are orbits under a crystallographic group. The two theorems were obtained by Delone, Dolbilin, Shtogrin and Galiulin well over 25 years ago (see [5]), although a proof of the Local Theorem for Tilings did not appear in print until Dolbilin \& Schattschneider [8]; see also Dolbilin, Lagarias and Senechal [9] for generalizations of the Local Theorem for Delone Sets.

In this paper we describe a local characterization of combinatorial tile-transitivity of monotypic tilings in $\mathbb{E}^{d}$; the result is the Local Theorem for Monotypic Tilings (see Theorem 3.1) proved in Section 3. This characterization is expressed in terms of combinatorial symmetry properties of large enough neighborhood complexes (coronas) of tiles. However, unlike in the original Local Theorem for Tilings, where the symmetries are induced by global isometries of the ambient space, the combinatorial symmetries are (at least, a priori) only defined on the neighborhood complexes (that is, locally).

In a sense, the new theorem sits between the Local Theorem for Tilings and the socalled Extension Theorem; the latter, in turn, is based on the Local Theorem for Tilings and Alexandrov's theorem in [1], and gives a criterion for a finite monohedral complex of polytopes to be extendable to a global isohedral tiling of space. See [6, 7] for a discussion and applications of the Extension Theorem. In the Extension Theorem, we begin with a finite monohedral complex, not with a global tiling, and then proceed by extending this finite complex to a global tiling by means of globally operating isometries of space. However, in the Local Theorem for Monotypic Tilings, we already have a global tiling and now must patch together global combinatorial isomorphisms from a given set of local isomorphisms. Note that the term "Extension Theorem" was used in Grünbaum \& Shephard [15] to refer to a different, albeit related, theorem.

\section{Basic notions and facts}

A tiling $\mathcal{T}$ of euclidean $d$-space $\mathbb{E}^{d}$ is a countable family of closed subsets of $\mathbb{E}^{d}$, the tiles of $\mathcal{T}$, which cover $\mathbb{E}^{d}$ without gaps and overlaps (see Grünbaum \& Shephard [15]). All tilings are taken to be locally finite, meaning that each point of $\mathbb{E}^{d}$ has a neighborhood that meets only finitely many tiles. We shall assume that the tiles of $\mathcal{T}$ are convex $d$ polytopes. (For a combinatorial analogue of the Local Theorem for Tilings it actually suffices to require the tiles to be homeomorphic images of convex polytopes; however, it is convenient to assume convexity. We shall elaborate on this in Remark 3.10.) A tiling $\mathcal{T}$ by convex $d$-polytopes is said to be face-to-face if the intersection of any two tiles is a 
face of each tile, possibly the empty face. For a face-to-face tiling $\mathcal{T}$, the set of all faces of tiles, ordered by inclusion, becomes a lattice when the entire space is adjoined as an improper maximal face of rank $d+1$ (see Stanley [22]); this is the face-lattice of $\mathcal{T}$ and is often identified with $\mathcal{T}$ (the improper face is usually ignored).

Our main interest is in locally finite face-to-face tilings which are monotypic. Let $T$ be a convex $d$-polytope. Recall that a tiling $\mathcal{T}$ of $\mathbb{E}^{d}$ is monotypic of type $T$ if each tile of $\mathcal{T}$ is a convex $d$-polytope combinatorially equivalent to $T$ (see $[3,16,20,21]$ ). The polytope $T$ is the combinatorial prototile of $\mathcal{T}$, and $T$ is said to admit the tiling $\mathcal{T}$. Monotypic tilings are combinatorial analogues of monohedral tilings, these being tilings in which each tile is congruent to a single tile.

A locally finite face-to-face tiling $\mathcal{T}$ of $\mathbb{E}^{d}$ is combinatorially tile-transitive if its combinatorial automorphism group $\Gamma(\mathcal{T})$ is transitive on the tiles. Such a tiling $\mathcal{T}$ must necessarily be monotypic. We mention in passing that combinatorial tile-transitivity is equivalent to topological tile-transitivity. (Recall that $\mathcal{T}$ is topologically tile-transitive if for any two tiles $P, Q$ of $\mathcal{T}$ there is a homeomorphism of $\mathbb{E}^{d}$ that maps $\mathcal{T}$ onto $\mathcal{T}$ and $P$ onto $Q$.) In fact, since the tiles are convex polytopes, each combinatorial automorphism of $\mathcal{T}$ can be realized by a homeomorphism of $\mathbb{E}^{d}$; moreover, this can be done in such a manner that the entire group $\Gamma(\mathcal{T})$ is realized by a group of homeomorphisms of $\mathbb{E}^{d}$. In other words, there is a group of homeomorphisms of $\mathbb{E}^{d}$ which is isomorphic to $\Gamma(\mathcal{T})$ and has the same action on the face-lattice of $\mathcal{T}$ as $\Gamma(\mathcal{T})$.

We frequently make use of the following lemma. Let $\mathcal{C}$ be a subcomplex of $\mathcal{T}$ such that every maximal face of $\mathcal{C}$ is a tile of $\mathcal{T}$; that is, every flag (maximal set of mutually incident faces) of $\mathcal{C}$ is also a flag of $\mathcal{T}$ (again we omit the improper face of $\mathcal{T}$ of rank $d+1)$. Recall that $\mathcal{C}$ is flag-connected if any two flags $\Phi$ and $\Psi$ of $\mathcal{C}$ can be joined by a finite sequence of flags

$$
\Phi=\Phi_{0}, \Phi_{1}, \ldots, \Phi_{n}=\Psi
$$

of $\mathcal{C}$ such that $\Phi_{j-1}$ and $\Phi_{j}$ differ by at most one face (that is, $\Phi_{j-1}$ and $\Phi_{j}$ are adjacent flags), for each $j=1, \ldots, n$; see, for example, [17, Sect.2A].

Lemma 2.1 Let $\mathcal{C}$ be a subcomplex of $\mathcal{T}$ such that every maximal face of $\mathcal{C}$ is a tile of $\mathcal{T}$. Let $\mathcal{C}$ be flag-connected, and let $\Phi$ be a flag of $\mathcal{C}$. Then every isomorphism $\alpha$ between $\mathcal{C}$ and a subcomplex of $\mathcal{T}$ is uniquely determined by its effect on $\Phi$.

Proof: Since every face of $\mathcal{C}$ is contained in a flag of $\mathcal{C}$ and every flag of $\mathcal{C}$ is also a flag of $\mathcal{T}$, it suffices to consider the action of $\alpha$ on the flags. Now let $\Psi$ be a flag of $\mathcal{C}$, and let $\Phi=\Phi_{0}, \Phi_{1}, \ldots, \Phi_{n}=\Psi$ be a sequence of flags of $\mathcal{C}$ such that $\Phi_{j-1}$ and $\Phi_{j}$ are adjacent for each $j$. Every isomorphism $\alpha$ preserves adjacency of flags; that is, $\alpha$ takes a pair of adjacent flags to a pair of adjacent flags. In particular, if $\Phi_{j-1}$ and $\Phi_{j}$ differ in their $i$-faces, then $\alpha\left(\Phi_{j-1}\right)$ and $\alpha\left(\Phi_{j}\right)$ also differ in their $i$-faces and hence $\alpha\left(\Phi_{j}\right)$ is uniquely determined by $\alpha\left(\Phi_{j-1}\right)$. It follows that $\alpha(\Psi)$ is uniquely determined by $\alpha(\Phi)$. This proves the lemma.

In a locally finite face-to-face tiling $\mathcal{T}$, any two tiles $P$ and $Q$ of $\mathcal{T}$ can be joined by a finite sequence of tiles

$$
P=P_{0}, P_{1}, \ldots, P_{n-1}, P_{n}=Q
$$


of $\mathcal{T}$ such that $P_{j-1} \cap P_{j}$ is a face of $P_{j-1}$ and $P_{j}$ of dimension at least $d-2$, for $j=1, \ldots, n$; we call $n$ the length of the sequence.

Definition 2.2 The minimum length of a sequence of tiles joining $P$ and $Q$ as in (2.1) is called the distance of $P$ and $Q$ in $\mathcal{T}$ and is denoted by $d(P, Q)$. (Note that consecutive tiles in any such sequence are supposed to intersect in faces of dimension at least $d-2$.

Specifically we are interested in sequences of tiles

$$
P=P_{0}, P_{1}, \ldots, P_{n-1}, P_{n}=Q
$$

of $\mathcal{T}$, in which $P_{j-1}$ and $P_{j}$ share a facet for $j=1, \ldots, n$. Any two tiles $P$ and $Q$ of $\mathcal{T}$ can be joined by such a sequence. In fact, the following more general statement is true; we include a proof for completeness.

Lemma 2.3 Let $\mathcal{T}$ be a locally finite face-to-face tiling of $E^{d}$ (or spherical or hyperbolic $d$-space) by convex d-polytopes, let $P$ and $Q$ be tiles of $\mathcal{T}$, and let $F$ be a face of $P$. Then $F$ is a face of $Q$ if and only if there exists a sequence of tiles

$$
P=P_{0}, P_{1}, \ldots, P_{n-1}, P_{n}=Q
$$

of $\mathcal{T}$, each containing $F$, such that $P_{j-1}$ and $P_{j}$ share a facet for $j=1, \ldots, n$.

Proof: One direction of the lemma is obvious. We prove the other direction for any locally finite face-to-face tiling $\mathcal{T}$ of a spherical, euclidean or hyperbolic $d$-space $\mathrm{X}^{\mathrm{d}}$. Note that the case $d=1$ is trivial. Now let $d \geq 2$ and assume inductively that the statement already holds for tilings of $\mathrm{X}^{\mathrm{j}}$ with $j \leq d-1$. Let $\mathcal{T}$ be a locally finite face-to-face tiling of $\mathrm{X}^{\mathrm{d}}$, let $P$ and $Q$ be tiles of $\mathcal{T}$, and let $F$ be a face of $P$ and $Q$ of dimension $k$ (say). Consider the star st $(F)$ of $F$ in $\mathcal{T}$, that is, the subcomplex of $\mathcal{T}$ consisting of the tiles of $\mathcal{T}$ which contain $F$, and their faces. Let $x$ be a relative interior point of $F$, and let $S$ be a small $(d-1)$-sphere centered at $x$ such that $S$ only intersects those faces of $\mathcal{T}$ which contain $F$. Then $S \cap F$ is a great $(k-1)$-sphere of $S$. Let $S^{\prime}$ be a great $(d-k-1)$-sphere of $S$ complementary to $S \cap F$ in $S$. Then $s t(F)$ induces a locally finite face-to-face tiling $\mathcal{T}^{\prime}$ on $S^{\prime}$ by spherical $(d-k-1)$-polytopes, such that the tiles of $\mathcal{T}^{\prime}$ are the intersections of $S^{\prime}$ with the tiles in $s t(F)$, and the faces of the tiles in $\mathcal{T}^{\prime}$ correspond to the faces of st $(F)$ containing $F$. In particular, $P^{\prime}:=P \cap S^{\prime}$ and $Q^{\prime}:=Q \cap S^{\prime}$ are tiles of $\mathcal{T}^{\prime}$. By the inductive hypothesis for $\mathcal{T}^{\prime}$ (applied with the empty face in place of $F$ ), there is a sequence of tiles

$$
P^{\prime}=P_{0}^{\prime}, P_{1}^{\prime}, \ldots, P_{n-1}^{\prime}, P_{n}^{\prime}=Q^{\prime}
$$

of $\mathcal{T}^{\prime}$ such that $P_{j-1}^{\prime}$ and $P_{j}^{\prime}$ have a facet in common for $j=1, \ldots, n$. Now, if $P=$ $P_{0}, P_{1}, \ldots, P_{n-1}, P_{n}=Q$ is the corresponding sequence of tiles contained in $s t(F)$, then each tile $P_{j}$ contains $F$ and any two consecutive tiles $P_{j-1}$ and $P_{j}$ meet again along a facet. This completes the proof.

Before we move on, observe that there are variants of the distance function of Definition 2.2 for the tiles of $\mathcal{T}$. They are obtained by requiring that any two consecutive tiles 
in (2.1) intersect in a face of dimension at least $l$, for a given $l \leq d-1$; the corresponding number $d_{l}(P, Q)$ is generally distinct from $d(P, Q)$ if $l \neq d-2$. In what follows we always take $l=d-2$; this corresponds to the original distance function $d(.,$.$) of Definition 2.2.$ (For arbitrary tilings which are not necessarily face-to-face, still another variant requires that any two consecutive tiles in (2.1) have non-empty intersection. However, we will not further discuss this here.)

Let $P$ be a tile of $\mathcal{T}$, and let $k \geq 0$ be an integer. The $k^{\text {th }}$ corona of $P$, denoted by $\mathcal{C}_{k}(P)$, is the subcomplex of $\mathcal{T}$ consisting of the tiles $Q$ of $\mathcal{T}$ with $d(P, Q) \leq k$, and their faces. In particular, the $0^{\text {th }}$ corona $\mathcal{C}_{0}(P)$ is the face-lattice of $P$ (consisting of $P$ and its faces), and the $1^{\text {st }}$ corona $\mathcal{C}_{1}(P)$ is the set of faces of tiles that intersect $P$ in a face of dimension at least $d-2$. More generally, if $k \geq 1$, then the $k^{t h}$ corona $\mathcal{C}_{k}(P)$ is the set of faces of tiles that intersect a tile in $\mathcal{C}_{k-1}(P)$ in a face of dimension at least $d-2$. Note that, by definition, a corona is a complex, not a set of tiles or a union of tiles; this differs from the use of the term in other articles, for example, in [8]. The term "corona" was introduced in [11] (but was used in a slightly different meaning).

It is possible for different tiles $P$ and $Q$ in a tiling to have the same corona, that is, $\mathcal{C}_{k}(P)=\mathcal{C}_{k}(Q)$ for some $k$ (and hence $\mathcal{C}_{j}(P)=\mathcal{C}_{j}(Q)$ for each $j \geq k$ ). Figure 1 depicts a patch of a plane tiling by triangles, in which two tiles $P$ and $Q$ have the same $1^{\text {st }}$ corona (see [19]).

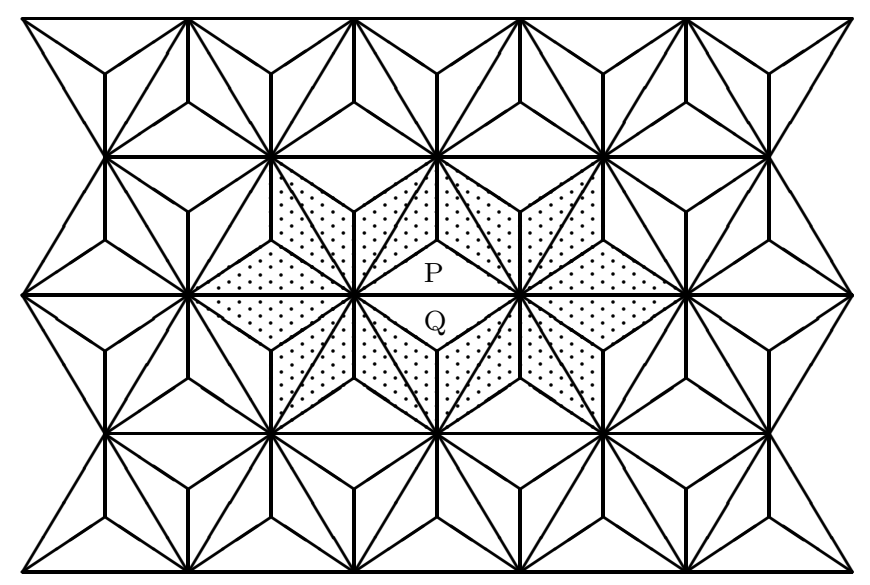

Figure 1: The tiles $P$ and $Q$ have the same $1^{\text {st }}$ corona. It consists of the dotted tiles as well as $P$ and $Q$, and their vertices and edges.

Therefore, in our considerations, it is important to distinguish coronas by their tile of reference. Accordingly, a centered $k^{\text {th }}$ corona is a pair $\left(P, \mathcal{C}_{k}(P)\right)$ consisting of a tile $P$ of $\mathcal{T}$, the center of the centered $k^{\text {th }}$ corona, and its $k^{\text {th }}$ corona $\mathcal{C}_{k}(P)$ in $\mathcal{T}$. We usually drop the center $P$ from the notation when it is clear from the context, that is, we simply denote $\left(P, \mathcal{C}_{k}(P)\right)$ by $\mathcal{C}_{k}(P)$.

Two centered $k^{\text {th }}$ coronas $\mathcal{C}_{k}(P)$ and $\mathcal{C}_{k}\left(P^{\prime}\right)$ of $\mathcal{T}$ are isomorphic if there exists an 
isomorphism of complexes $\alpha: \mathcal{C}_{k}(P) \rightarrow \mathcal{C}_{k}\left(P^{\prime}\right)$ with $\alpha(P)=P^{\prime}$; such a map $\alpha$ is called an isomorphism of centered $k^{\text {th }}$ coronas. In this situation, since $\alpha$ maps $P$ to $P^{\prime}$, it also preserves distances from the centers and thus restricts to an isomorphism $\alpha: \mathcal{C}_{j}(P) \rightarrow$ $\mathcal{C}_{j}\left(P^{\prime}\right)$ of centered $j^{\text {th }}$ coronas for each $j \leq k$. Similarly, any automorphism $\alpha$ of the whole tiling $\mathcal{T}$ that maps $P$ to $P^{\prime}$ restricts to an isomorphism $\alpha: \mathcal{C}_{j}(P) \rightarrow \mathcal{C}_{j}\left(P^{\prime}\right)$ of centered $j^{\text {th }}$ coronas for each $j \geq 0$.

If $\mathcal{C}_{k}(P)$ is a centered $k^{t h}$ corona, we denote by $\Gamma\left(\mathcal{C}_{k}(P)\right)$ its group of automorphisms; once again, by definition, each such automorphism fixes the center $P$. (In other words, this group is the stabilizer of the center in the full automorphism group of the corresponding "non-centered" corona.)

The automorphism groups of centered coronas at increasing levels $k$ are related. In particular, if $P$ is a tile of $\mathcal{T}$, then we have the following infinite chain of subgroup relationships,

$$
\Gamma_{P}(\mathcal{T}) \subseteq \ldots \subseteq \Gamma\left(\mathcal{C}_{k}(P)\right) \subseteq \Gamma\left(\mathcal{C}_{k-1}(P)\right) \subseteq \ldots \subseteq \Gamma\left(\mathcal{C}_{1}(P)\right) \subseteq \Gamma\left(\mathcal{C}_{0}(P)\right)=\Gamma(P),
$$

with the stabilizer $\Gamma_{P}(\mathcal{T})$ of $P$ in $\Gamma(\mathcal{T})$ on the left and the combinatorial automorphism group $\Gamma(P)$ of $P$ on the right. In fact, if $k \geq 1$, then every automorphism of $\mathcal{C}_{k}(P)$ restricts to an automorphism of $\mathcal{C}_{k-1}(P)$ and is uniquely determined by its effect on $\mathcal{C}_{k-1}(P)$; note that, since $\mathcal{C}_{k-1}(P)$ contains a flag and $\mathcal{C}_{k}(P)$ is flag-connected, the latter follows from Lemma 2.1. Similarly, if $k \geq 0$, then every automorphism of $\mathcal{T}$ that fixes $P$ restricts to an automorphism of $\mathcal{C}_{k}(P)$ and is uniquely determined by this restriction. Note that $\Gamma(P)$ is a finite group, so there can only be a finite number of proper ascents in (2.2).

\section{The Local Theorem for Monotypic Tilings}

The following Local Theorem for Monotypic Tilings is a combinatorial analogue of the Local Theorem for Tilings (see Section 4).

Theorem 3.1 Let $\mathcal{T}$ be a locally finite face-to-face tiling of $\mathbb{E}^{d}$ by convex polytopes. Then $\mathcal{T}$ is combinatorially tile-transitive if and only if there exists a positive integer $k$ with the following properties:

1. Any two centered $k^{\text {th }}$ coronas of $\mathcal{T}$ are isomorphic (as centered coronas).

2. $\Gamma\left(\mathcal{C}_{k}(P)\right)=\Gamma\left(\mathcal{C}_{k-1}(P)\right)$ for each tile $P$ of $\mathcal{T}$.

Moreover, in this case, $\Gamma\left(\mathcal{C}_{k}(P)\right)=\Gamma_{P}(\mathcal{T})$.

Before proceeding with the proof, we illustrate by way of an example that the second condition of Theorem 3.1 is essential and cannot be ignored. Consider plane tilings $\mathcal{T}$ by quadrilaterals, in which each tile has one vertex of valence 3 and three vertices of valence 5 ; that is, $\mathcal{T}$ is a homogeneous tiling of type $\left[3.5^{3}\right]$ (see [15]). It follows from the results of Grünbaum \& Shephard [13, Thm.4.8, Fig.4.4] that such tilings $\mathcal{T}$ cannot be combinatorially tile-transitive (that is, $\mathcal{T}$ cannot be homeohedral). Clearly, the 1 -st coronas of $\mathcal{T}$ are 
mutually isomorphic; that is, $\mathcal{T}$ satisfies the first condition of Theorem 3.1 with $k=1$. However, $\mathcal{T}$ fails to satisfy the second condition with $k=1$, so Theorem 3.1 will not allow the conclusion that $\mathcal{T}$ is combinatorially tile-transitive. In fact, the automorphism groups of the 0 -th corona and the 1 -st corona of a tile $P$ are not the same. The 0 -th corona of $P$ consists of $P$ and its faces, and its automorphism group is the dihedral group of order 8 . On the other hand, every automorphism of the centered 1-st corona of $P$ must necessarily fix the 3 -valent vertex of $P$; however, there are only two such automorphisms. Note that [13] also discusses more general classes of tilings with similar properties.

Proof of Theorem 3.1: First note that, because of the first condition, the second could be replaced by the weaker condition requiring only that the two consecutive groups coincide for at least one tile, not all tiles, $P$.

Now suppose that $\Gamma(\mathcal{T})$ is combinatorially tile-transitive. If $P$ and $P^{\prime}$ are tiles of $\mathcal{T}$, then every element $\gamma \in \Gamma(\mathcal{T})$ that maps $P$ to $P^{\prime}$ necessarily induces an isomorphism of centered coronas between the centered $k^{t h}$ coronas of $P$ and $P^{\prime}$, for each $k \geq 0$; thus the first condition is met for every integer $k \geq 0$. Moreover, we have

$$
\Gamma\left(\mathcal{C}_{j}\left(P^{\prime}\right)\right)=\gamma \Gamma\left(\mathcal{C}_{j}(P)\right) \gamma^{-1}
$$

for each $j \geq 0$, so that an integer $k$ that satisfies the second condition for $P$ will also satisfy it for $P^{\prime}$; thus $k$ will not depend on the tile. But if $P$ is a tile of $\mathcal{T}$, then it is a polytope with a finite group $\Gamma(P)$, so an infinite chain of subgroups of $\Gamma(P)$ must necessarily stutter. Hence, in the infinite chain of (2.2), there must be a pair of consecutive subgroups, $\Gamma\left(\mathcal{C}_{k}(P)\right)$ and $\Gamma\left(\mathcal{C}_{k-1}(P)\right)$ for some positive integer $k$ (say), which coincide. This proves that the two conditions of the theorem are necessary.

The proof of sufficiency is more complicated. Let $k$ be a positive integer satisfying the two conditions of the theorem. We shall describe how a local isomorphism of centered $k^{t h}$ coronas can be extended step by step to an isomorphism of the whole tiling $\mathcal{T}$, thereby becoming a global isomorphism. More specifically, let $P$ and $P^{\prime}$ be tiles of $\mathcal{T}$. Then, by the first condition of the theorem, there exists an isomorphism of centered $k^{\text {th }}$ coronas $\alpha$ : $\mathcal{C}_{k}(P) \rightarrow \mathcal{C}_{k}\left(P^{\prime}\right)$; in particular, $\alpha(P)=P^{\prime}$. We will prove that $\alpha$ induces an automorphism of $\mathcal{T}$ which moves $P$ to $P^{\prime}$.

We break the sufficiency proof into a series of lemmas which accomplish the following steps.

1. Every isomorphism of two centered $(k-1)^{s t}$ coronas of $\mathcal{T}$ extends uniquely to an isomorphism of the corresponding two centered $k^{\text {th }}$ coronas (see Lemma 3.2).

2. Every isomorphism of two centered $k^{t h}$ coronas $\alpha$ extends uniquely to neighboring centered $k^{t h}$ coronas (see Lemma 3.3). More precisely, if $\alpha: \mathcal{C}_{k}(P) \rightarrow \mathcal{C}_{k}\left(P^{\prime}\right)$ is given and $Q$ is a tile with $d(P, Q)=1$, then there exists a unique isomorphism of centered $k^{\text {th }}$ coronas $\beta: \mathcal{C}_{k}(Q) \rightarrow \mathcal{C}_{k}\left(Q^{\prime}\right)$ such that $\alpha$ and $\beta$ coincide on both $\mathcal{C}_{k-1}(P)$ and $\mathcal{C}_{k-1}(Q)$; necessarily, $Q^{\prime}=\alpha(Q)$.

3. Every isomorphism of two centered $k^{\text {th }}$ coronas $\alpha$ extends uniquely along sequences of tiles in which any two consecutive tiles share a facet (see Lemmas 3.4 and 3.5). 
More precisely, if $P=P_{0}, P_{1}, \ldots, P_{n-1}, P_{n}=Q$ is such a sequence connecting two tiles $P$ and $Q$, then $\alpha: \mathcal{C}_{k}(P) \rightarrow \mathcal{C}_{k}\left(P^{\prime}\right)$ induces uniquely an isomorphism of centered $k^{\text {th }}$ coronas $\beta: \mathcal{C}_{k}(Q) \rightarrow \mathcal{C}_{k}\left(Q^{\prime}\right)$ determined by a sequence of isomorphisms of centered $k^{\text {th }}$ coronas $\alpha=\beta_{0}, \beta_{1}, \ldots, \beta_{n-1}, \beta_{n}=\beta$, with $\beta_{i}: \mathcal{C}_{k}\left(P_{i}\right) \rightarrow \mathcal{C}_{k}\left(P_{i}^{\prime}\right)$ for some $P_{i}^{\prime}$. In particular, $\beta$ does not depend on the original sequence of tiles chosen to connect $P$ and $Q$.

4. Every isomorphism of two centered $k^{\text {th }}$ coronas $\alpha$ induces a global automorphism of $\mathcal{T}$ (see Lemmas 3.5 and 3.6). More precisely, if $\alpha: \mathcal{C}_{k}(P) \rightarrow \mathcal{C}_{k}\left(P^{\prime}\right)$ is extended in this fashion along sequences of tiles throughout $\mathcal{T}$, then each resulting isomorphism of centered $k^{t h}$ coronas $\beta: \mathcal{C}_{k}(Q) \rightarrow \mathcal{C}_{k}\left(Q^{\prime}\right)$ restricts faithfully to a local mapping $\alpha_{Q}$ between the face lattices of $Q$ and $Q^{\prime}$, and all these local mappings fit together coherently to determine an extension of $\alpha$ to a global automorphism of $\mathcal{T}$.

For the following lemmas bear in mind that $k$ is always a positive integer satisfying the two conditions of the theorem.

Lemma 3.2 Let $P, P^{\prime}$ be tiles of $\mathcal{T}$, and let $\bar{\alpha}: \mathcal{C}_{k-1}(P) \rightarrow \mathcal{C}_{k-1}\left(P^{\prime}\right)$ be an isomorphism of centered $(k-1)^{\text {st }}$ coronas. Then there exists a unique isomorphism of centered $k^{\text {th }}$ coronas $\alpha: \mathcal{C}_{k}(P) \rightarrow \mathcal{C}_{k}\left(P^{\prime}\right)$ which extends $\bar{\alpha}$, that is, $\left.\alpha\right|_{\mathcal{C}_{k-1}(P)}=\bar{\alpha}$.

Proof: First observe that every automorphism of the $(k-1)^{\text {st }}$ corona $\mathcal{C}_{k-1}(P)$ extends uniquely to an automorphism of the $k^{t h}$ corona $\mathcal{C}_{k}(P)$. In fact, $\Gamma\left(\mathcal{C}_{k}(P)\right)=\Gamma\left(\mathcal{C}_{k-1}(P)\right)$ and $\mathcal{C}_{k}(P)$ is flag-connected, so every element $\bar{\gamma} \in \Gamma\left(\mathcal{C}_{k-1}(P)\right)$ uniquely determines an element $\gamma \in \Gamma\left(\mathcal{C}_{k}(P)\right)$ such that $\left.\gamma\right|_{\mathcal{C}_{k-1}(P)}=\bar{\gamma}$ (see Lemma 2.1).

Now let $\alpha: \mathcal{C}_{k}(P) \rightarrow \mathcal{C}_{k}\left(P^{\prime}\right)$ be any isomorphism of centered $k^{\text {th }}$ coronas; by assumption such isomorphisms exist. Then $\alpha$ restricts to an isomorphism of centered $(k-1)^{s t}$ coronas, and

$$
\bar{\gamma}:=\left.\alpha^{-1}\right|_{\mathcal{C}_{k-1}\left(P^{\prime}\right)} \bar{\alpha}: \mathcal{C}_{k-1}(P) \rightarrow \mathcal{C}_{k-1}(P)
$$

is an automorphism of $\mathcal{C}_{k-1}(P)$. In particular,

$$
\left.\alpha\right|_{\mathcal{C}_{k-1}(P)} \bar{\gamma}=\bar{\alpha}
$$

If $\gamma$ is the extension of $\bar{\gamma}$ to $\mathcal{C}_{k}(P)$, then the isomorphism of centered $k^{\text {th }}$ coronas $\alpha \gamma$ : $\mathcal{C}_{k}(P) \rightarrow \mathcal{C}_{k}\left(P^{\prime}\right)$ satisfies

$$
\left.(\alpha \gamma)\right|_{\mathcal{C}_{k-1}(P)}=\left.\alpha\right|_{\mathcal{C}_{k-1}(P)} \bar{\gamma}=\bar{\alpha}
$$

Thus $\alpha \gamma$ is an extension of $\bar{\alpha}$, and is unique, by the uniqueness of $\gamma$. Now the lemma is immediate if we replace the original $\alpha$ by $\alpha \gamma$.

Lemma 3.3 Let $P, P^{\prime}$ be tiles of $\mathcal{T}$, let $\alpha: \mathcal{C}_{k}(P) \rightarrow \mathcal{C}_{k}\left(P^{\prime}\right)$ be an isomorphism of centered $k^{\text {th }}$ coronas, and let $Q$ be a tile with $d(P, Q)=1$. Then there exists a unique isomorphism of centered $k^{\text {th }}$ coronas $\beta: \mathcal{C}_{k}(Q) \rightarrow \mathcal{C}_{k}\left(Q^{\prime}\right)$, with $Q^{\prime}=\alpha(Q)$, such that

$$
\left.\alpha\right|_{\mathcal{C}_{k-1}(Q)}=\left.\beta\right|_{\mathcal{C}_{k-1}(Q)} \quad \text { and }\left.\quad \alpha\right|_{\mathcal{C}_{k-1}(P)}=\left.\beta\right|_{\mathcal{C}_{k-1}(P)} .
$$


Proof: First observe that the lemma only claims that $\alpha$ and $\beta$ agree on the centered $(k-1)^{s t}$ coronas $\mathcal{C}_{k-1}(P)$ and $\mathcal{C}_{k-1}(Q)$, but not also on the (larger) intersection of the corresponding $k^{t h}$ coronas $\mathcal{C}_{k}(P)$ and $\mathcal{C}_{k}(Q)$. (However, the latter will follow once the theorem has been proved.)

Let $Q^{\prime}:=\alpha(Q)$. Clearly, $d\left(P^{\prime}, Q^{\prime}\right)=1$. Then the restricted mapping $\left.\alpha\right|_{\mathcal{C}_{k-1}(Q)}$ is an isomorphism of centered $(k-1)^{s t}$ coronas between $\mathcal{C}_{k-1}(Q)$ and $\mathcal{C}_{k-1}\left(Q^{\prime}\right)$. By Lemma 3.2, it has a unique extension to an isomorphism of centered $k^{\text {th }}$ coronas $\beta: \mathcal{C}_{k}(Q) \rightarrow \mathcal{C}_{k}\left(Q^{\prime}\right)$, so in particular we have $\left.\alpha\right|_{\mathcal{C}_{k-1}(Q)}=\left.\beta\right|_{\mathcal{C}_{k-1}(Q)}$.

We now prove that the relationship between $\alpha$ and $\beta$ is symmetric. In fact, if $k \geq 2$, then $\mathcal{C}_{k-2}(P) \subseteq \mathcal{C}_{k-1}(Q)$, so we can directly appeal to Lemma 2.1 using that $\left.\alpha\right|_{\mathcal{C}_{k-2}(P)}=$ $\left.\beta\right|_{\mathcal{C}_{k-2}(P)}$. However, the argument is more complicated if $k=1$. First we make the following general observation, which is valid for any $k \geq 1$.

If $G, H$ are tiles of $\mathcal{T}$ contained in $\mathcal{C}_{k}(P) \cap \mathcal{C}_{k}(Q)$ such that $G \cap H$ is a facet and $\left.\alpha\right|_{\mathcal{C}_{0}(G)}=\left.\beta\right|_{\mathcal{C}_{0}(G)}$, then also

$$
\left.\alpha\right|_{\mathcal{C}_{0}(H)}=\left.\beta\right|_{\mathcal{C}_{0}(H)}
$$

Notice that $\alpha(H), \beta(H)$ each must meet $\alpha(G)=\beta(G)$ in the common facet $\alpha(G \cap H)=$ $\beta(G \cap H)$, so they must actually be the same tiles; but since $\alpha$ and $\beta$ already coincide on each face of $G \cap H$, this then implies that $\left.\alpha\right|_{\mathcal{C}_{0}(H)}=\left.\beta\right|_{\mathcal{C}_{0}(H)}$.

We now complete the argument as follows. Since $d(P, Q)=1$, the tiles $P$ and $Q$ intersect in a face of dimension at least $d-2$. If $P \cap Q$ is a facet and again $k=1$, then the above argument (applied with $G=Q$ and $H=P$ ) shows that $\alpha$ and $\beta$ coincide on $\mathcal{C}_{0}(P)=\mathcal{C}_{k-1}(P)$, as claimed. On the other hand, if $P \cap Q$ is a $(d-2)$-face, then there exists a sequence of tiles

$$
Q=Q_{0}, Q_{1}, \ldots, Q_{m-1}, Q_{m}=P
$$

each containing $P \cap Q$, such that $Q_{j-1} \cap Q_{j}$ is a common facet of $Q_{j-1}$ and $Q_{j}$ for $j=1, \ldots, m$. We now apply the same argument as before to the pairs of consecutive tiles in this sequence, beginning with $Q=Q_{0}, Q_{1}$, and successively moving from $Q_{j-1}, Q_{j}$ to $Q_{j}, Q_{j+1}$ until we reach $Q_{m-1}, Q_{m}=P$. Then, at this final stage, we can conclude that $\alpha$ and $\beta$ coincide on $\mathcal{C}_{0}(P)=\mathcal{C}_{k-1}(P)$.

In summary, we now know that every isomorphism of centered $k^{\text {th }}$ coronas extends uniquely to neighboring centered $k^{\text {th }}$ coronas, in the sense that each new mapping agrees with the original isomorphism on at least the two corresponding centered $(k-1)^{s t}$ coronas.

We now exploit the simply-connectedness of the underlying space to further extend such isomorphisms. Once again, let $P$ and $P^{\prime}$ be tiles of $\mathcal{T}$, and let $\alpha: \mathcal{C}_{k}(P) \rightarrow \mathcal{C}_{k}\left(P^{\prime}\right)$ be an isomorphism of centered $k^{\text {th }}$ coronas. Let $Q$ be any tile of $\mathcal{T}$, not necessarily with $d(P, Q)=1$. We shall extend $\alpha$ along finite sequences of tiles

$$
P=P_{0}, P_{1}, \ldots, P_{n-1}, P_{n}=Q,
$$

where $P_{j-1} \cap P_{j}$ is a facet of $P_{j-1}$ and $P_{j}$, and hence $d\left(P_{j-1}, P_{j}\right)=1$, for each $j=1, \ldots, n$. 
Lemma 3.4 Let $P=P_{0}, P_{1}, \ldots, P_{n-1}, P_{n}=Q$ be a finite sequence of tiles as in (3.2), let $P^{\prime}$ be a tile of $\mathcal{T}$, and let $\alpha: \mathcal{C}_{k}(P) \rightarrow \mathcal{C}_{k}\left(P^{\prime}\right)$ be an isomorphism of centered $k^{\text {th }}$ coronas. Then $\alpha$ admits a unique extension along the sequence to an isomorphism of centered $k^{\text {th }}$ coronas

$$
\beta: \mathcal{C}_{k}(Q) \rightarrow \mathcal{C}_{k}\left(Q^{\prime}\right)
$$

with $Q^{\prime}$ a tile.

Proof: We repeatedly apply Lemma 3.3 using that any two consecutive tiles in the sequence are at distance 1 . Then we obtain a sequence of isomorphisms of centered $k^{\text {th }}$ coronas

$$
\alpha=: \beta_{0}, \beta_{1}, \ldots, \beta_{n-1}, \beta_{n}=: \beta,
$$

where $\beta_{j}: \mathcal{C}_{k}\left(P_{j}\right) \longrightarrow \mathcal{C}_{k}\left(P_{j}^{\prime}\right)$ for $j=0,1, \ldots, n$, with $P_{0}^{\prime}=P^{\prime}$ and $P_{j}^{\prime}=\beta_{j-1}\left(P_{j}\right)$ for $j \geq 1$. In particular, $\beta$ is an isomorphism between the centered $k^{\text {th }}$ corona of $Q$ and the centered $k^{t h}$ corona of $Q^{\prime}:=P_{n}^{\prime}$. At each stage $j$, the extension of $\beta_{j-1}$ to $\beta_{j}$ is unique, hence $\beta$ is uniquely determined by $\alpha$ and the given sequence of tiles.

In the next lemma we show that the extension $\beta$ of $\alpha$ as in Lemma 3.4 does not actually depend on the sequence of tiles joining $P$ to $Q$. Suppose we have two such sequences of tiles,

$$
P=P_{0}, P_{1}, \ldots, P_{n-1}, P_{n}=Q
$$

and

$$
P=R_{0}, R_{1}, \ldots, R_{m-1}, R_{m}=Q
$$

(say), where again consecutive tiles in a sequence intersect in facets. Consider the dual edge graph $\mathcal{G}$ of $\mathcal{T}$; this is a graph in $\mathbb{E}^{d}$ whose nodes are the barycenters of the tiles in $\mathcal{T}$ and whose arcs ("edges") connect the barycenters of tiles that share a common facet. The sequences of tiles which join $P$ and $Q$ and in which consecutive tiles meet along facets all correspond to paths along the edges of $\mathcal{G}$ that start at the barycenter of $P$ and end at the barycenter of $Q$. Now, since the underlying space $\mathbb{E}^{d}$ is simply-connected, the two paths associated with the two sequences joining $P$ and $Q$ are homotopic and can be moved into each other by a homotopy that passes only over $(d-2)$-faces of $\mathcal{T}$ (that is, it never passes over faces of dimension less than $d-2)$. Each time the homotopy passes over a $(d-2)$-face $F$ (say), the corresponding sequence of tiles changes in such a way that its string of tiles containing $F$ is replaced by a new (complementary) string of tiles containing $F$, such that the two strings together completely surround $F$ in $\mathcal{T}$ and begin with the same tiles and end with the same tiles. Therefore it suffices to show that the extension of $\alpha$ to the $k^{\text {th }}$ corona of $Q$ does not depend on local changes (standard elementary moves) of this kind in a sequence.

Lemma 3.5 Let $P, P^{\prime}, Q, Q^{\prime}$ be tiles of $\mathcal{T}$, let $\alpha: \mathcal{C}_{k}(P) \rightarrow \mathcal{C}_{k}\left(P^{\prime}\right)$ and $\beta: \mathcal{C}_{k}(Q) \rightarrow$ $\mathcal{C}_{k}\left(Q^{\prime}\right)$ be isomorphisms of centered $k^{\text {th }}$ coronas, and let $\beta$ be obtained as in Lemma 3.4 by extending $\alpha$ along a sequence of tiles connecting $P$ and $Q$ as in (3.2). Then $\beta$ does not depend on the particular choice of sequence of tiles. 
Proof: $\quad$ Let $F$ be a $(d-2)$-face of $\mathcal{T}$. We consider sequences with tiles from $s t(F)$ (the star of $F$ ), where again any two consecutive tiles in a sequence meet in a facet. First we explain why it is sufficient to consider closed sequences of tiles and their corresponding sequences of isomorphisms.

Clearly, if $R$ is a tile in $s t(F)$, then any two sequences of tiles which connect $R$ to another tile $S$ in $s t(F)$ with tiles from $s t(F)$, yield, in an obvious way, a "closed" sequence of tiles from $s t(F)$ which begins and ends at $R$ and contains $S$, and vice versa. A similar statement is also true for sequences of isomorphisms of centered $k^{\text {th }}$ coronas. In fact, if $R, S$ are tiles in $s t(F)$ and $\sigma: \mathcal{C}_{k}(S) \rightarrow \mathcal{C}_{k}\left(S^{\prime}\right)$ is an isomorphism of centered $k^{t h}$ coronas, then any two sequences of isomorphisms of centered $k^{t h}$ coronas which extend $\sigma$ to $\mathcal{C}_{k}(R)$ along two sequences of tiles which connect $S$ to $R$ in $s t(F)$, determine a sequence of isomorphisms of centered $k^{\text {th }}$ coronas which begins and ends with isomorphisms defined on $\mathcal{C}_{k}(R)$, and contains $\sigma$, and vice versa. Here we are using the fact, established in Lemma 3.3, that the relationship of one isomorphism being the extension of another to a neighboring corona is symmetric. Our task is to show that this new sequence of isomorphisms is in fact "closed", meaning that it begins and ends with the same isomorphism on $\mathcal{C}_{k}(R)$.

Now let $R=R_{0}, R_{1}, \ldots, R_{l-1}, R_{l}=R$ be a closed sequence of tiles from $s t(F)$, let $\gamma: \mathcal{C}_{k}(R) \rightarrow \mathcal{C}_{k}\left(R^{\prime}\right)$ be an isomorphism of centered $k^{t h}$ coronas, and let

$$
\gamma=: \gamma_{0}, \gamma_{1}, \ldots, \gamma_{l-1}, \gamma_{l}=: \delta
$$

be the corresponding sequence of isomorphisms of centered $k^{\text {th }}$ coronas which extends $\gamma$ along the sequence of tiles, where $\gamma_{j}: \mathcal{C}_{k}\left(R_{j}\right) \longrightarrow \mathcal{C}_{k}\left(R_{j}^{\prime}\right)$ for $j=0,1, \ldots, l$, with $R_{0}^{\prime}=R^{\prime}$ and $R_{j}^{\prime}=\beta_{j-1}\left(R_{j}\right)$ for $j \geq 1$. We need to show that $\delta=\gamma$.

Once again we appeal to the symmetry in Lemma 3.3. For each $j \geq 1$, the isomorphisms $\gamma_{j-1}$ and $\gamma_{j}$ agree on the $(k-1)^{s t}$ centered coronas $\mathcal{C}_{k-1}\left(R_{j-1}\right)$ and $\mathcal{C}_{k-1}\left(R_{j}\right)$. In particular, when $k \geq 2$, the $(k-2)^{n d}$ corona $\mathcal{C}_{k-2}(R)$ is contained in $\mathcal{C}_{k-1}\left(R_{j}\right)$ for each $j \geq 0$, so the isomorphisms $\gamma_{j}$ certainly all agree on $\mathcal{C}_{k-2}(R)$; in this case, $\delta$ and $\gamma$ also agree on $\mathcal{C}_{k-2}(R)$, and hence $\delta=\gamma$ by Lemma 2.1. However, this argument does not apply if $k=1$. For general $k$ we can argue as follows.

We prove by induction on $j$ that

$$
\left.\gamma_{j}\right|_{\mathcal{C}_{0}\left(R_{i}\right)}=\left.\gamma_{0}\right|_{\mathcal{C}_{0}\left(R_{i}\right)} \quad(0 \leq i \leq j) .
$$

In particular, when $j=l$, we see that $\delta$ and $\gamma$ agree on $\mathcal{C}_{0}(R)$, and hence $\delta=\gamma$ by Lemma 2.1.

To begin the induction, first notice that the property holds for $j=1$. Suppose $j \geq 2$. For the inductive step we may now assume the following: $\gamma_{j-1}$ and $\gamma_{0}$ agree on each $\mathcal{C}_{0}\left(R_{i}\right)$ with $0 \leq i \leq j-1 ; \gamma_{j}$ and $\gamma_{1}$ agree on each $\mathcal{C}_{0}\left(R_{i}\right)$ with $1 \leq i \leq j$; and $\gamma_{j-1}$ and $\gamma_{1}$ agree on each $\mathcal{C}_{0}\left(R_{i}\right)$ with $1 \leq i \leq j-1$. Here the second and third properties are obtained from the inductive hypothesis for $j-1$ and $j-2$, respectively, applied with $\gamma_{1}$ as start of the sequence (in place of $\gamma_{0}$ ). These three assumptions already imply that $\gamma_{j}$ and $\gamma_{0}$ agree on each $\mathcal{C}_{0}\left(R_{i}\right)$ with $1 \leq i \leq j-1$, so only the two cases $i=0$ and $i=j$ need 
checking. Now, when $i=0$, observe that $\gamma_{j}\left(R_{0}\right)$ and $\gamma_{0}\left(R_{0}\right)$ each is a tile adjacent to the tile $\gamma_{j}\left(R_{1}\right)=\gamma_{0}\left(R_{1}\right)$ along the common facet $\gamma_{j}\left(R_{0} \cap R_{1}\right)=\gamma_{0}\left(R_{0} \cap R_{1}\right)$. However, $\gamma_{j}$ and $\gamma_{0}$ are isomorphisms of centered $k^{\text {th }}$ coronas, so their images of $R_{0}$ and $R_{1}$ certainly are distinct. Hence we must have $\gamma_{j}\left(R_{0}\right)=\gamma_{0}\left(R_{0}\right)$. On the other hand, we know that $\gamma_{j}$ and $\gamma_{0}$ agree on the face-lattice of the $(d-1)$-polytope $R_{0} \cap R_{1}$, so $\gamma_{j}$ and $\gamma_{0}$ must also agree on the face-lattice $\mathcal{C}_{0}\left(R_{0}\right)$ of $R_{0}$. This settles the case $i=0$. The case $i=j$ can be dealt with in a similar way using the inductive assumption and the adjacency of $R_{j}$ to $R_{j-1}$ along the common facet $R_{j-1} \cap R_{j}$. This completes the proof by induction.

In conclusion, the above considerations prove that a single elementary move in a sequence of tiles does not affect the extension. Therefore, the extension of $\alpha$ to $\beta$ along a sequence of tiles as in (3.2) does not depend on the actual sequence. This completes the proof.

At this stage it is convenient to concentrate on the action of isomorphisms on the $0^{\text {th }}$ coronas of tiles, that is, on the face-lattices of tiles. In particular, if an isomorphism of centered $k^{t h}$ coronas $\alpha: \mathcal{C}_{k}(P) \rightarrow \mathcal{C}_{k}\left(P^{\prime}\right)$ is extended along sequences of tiles which connect $P$ to other tiles $Q$ as in (3.2), then the resulting isomorphisms of centered $k^{t h}$ coronas $\beta: \mathcal{C}_{k}(Q) \rightarrow \mathcal{C}_{k}\left(Q^{\prime}\right)$ restrict faithfully to isomorphisms

$$
\alpha_{Q}: \mathcal{C}_{0}(Q) \rightarrow \mathcal{C}_{0}\left(Q^{\prime}\right)
$$

that is, $\alpha_{Q}:=\left.\beta\right|_{\mathcal{C}_{0}(Q)}$. Note again that $\alpha_{Q}$ does not depend on the particular choice of sequence of tiles employed to obtain $\beta$ from $\alpha$.

The following lemma implies that we can recover $\beta$ from the restricted mappings, so information is not lost in this process.

Lemma 3.6 Let $P, P^{\prime}, Q, Q^{\prime}$ be tiles of $\mathcal{T}$, let $\alpha: \mathcal{C}_{k}(P) \rightarrow \mathcal{C}_{k}\left(P^{\prime}\right)$ and $\beta: \mathcal{C}_{k}(Q) \rightarrow$ $\mathcal{C}_{k}\left(Q^{\prime}\right)$ be isomorphisms of centered $k^{\text {th }}$ coronas, and let $\beta$ be obtained as in Lemma 3.4 by extending $\alpha$ along a sequence of tiles connecting $P$ and $Q$ as in (3.2). If $R$ is a tile from $\mathcal{C}_{k}(Q)$ and $\alpha_{R}$ the corresponding isomorphism on $\mathcal{C}_{0}(R)$ obtained as in (3.4), then $\alpha_{R}$ and $\beta$ agree on $\mathcal{C}_{0}(R)$, that is, $\alpha_{R}=\left.\beta\right|_{\mathcal{C}_{0}(R)}$.

Proof: As $\alpha_{R}$ does not depend on the particular sequence of tiles chosen to link $P$ to $R$, we can take a suitable sequence passing through $Q$. First, since $r:=d(Q, R) \leq k$, there is a sequence $Q=Q_{0}, Q_{1}, \ldots, Q_{r}=R$ of tiles in $\mathcal{C}_{k}(Q)$ such that $d\left(Q_{i-1}, Q_{i}\right)=1$ for $i \geq 1$ and hence $d\left(Q, Q_{i}\right)=i$ for $i \geq 0$. Now, if a pair of consecutive tiles $Q_{i-1}, Q_{i}$ intersects in a $(d-2)$-face (but not a facet), then replace this pair in the sequence by a string of tiles

$$
Q_{i-1}=Q_{i-1}^{0}, Q_{i-1}^{1}, \ldots, Q_{i-1}^{j_{i}-1}, Q_{i-1}^{j_{i}}=Q_{i},
$$

each containing this $(d-2)$-face, such that $Q_{i-1}^{j-1} \cap Q_{i-1}^{j}$ is a common facet of $Q_{i-1}^{j-1}$ and $Q_{i-1}^{j}$ for $j=1, \ldots, j_{i}$. Finally, choose a sequence which connects $P$ and $Q$ as in (3.2), and then concatenate it with the sequence of $Q_{i}$ 's and $Q_{i}^{j}$ 's to produce a sequence of tiles which joins $P$ to $R$ via $Q$. By construction, any two consecutive tiles in this sequence meet along a facet. 
Now consider the corresponding sequence of isomorphisms of centered $k^{\text {th }}$ coronas which extends $\alpha$, via $\beta$, to an isomorphism of centered $k^{t h}$ coronas $\gamma: \mathcal{C}_{k}(R) \rightarrow \mathcal{C}_{k}\left(R^{\prime}\right)$. Let $\beta_{i}: \mathcal{C}_{k}\left(Q_{i}\right) \rightarrow \mathcal{C}_{k}\left(Q_{i}^{\prime}\right)$ denote the isomorphism corresponding to $Q_{i}$, for $i=0,1, \ldots, r$. If the tiles $Q_{i-1}$ and $Q_{i}$ share a facet (and thus no string of tiles was inserted between them), then Lemma 3.3 directly implies that $\beta_{i-1}$ and $\beta_{i}$ agree on $\mathcal{C}_{k-1}\left(Q_{i-1}\right)$ and $\mathcal{C}_{k-1}\left(Q_{i}\right)$. On the other hand, if

$$
\beta_{i-1}=\beta_{i-1}^{0}, \beta_{i-1}^{1}, \ldots, \beta_{i-1}^{j_{i}-1}, \beta_{j-1}^{j_{i}}=\beta_{i}
$$

(say) corresponds to a string of tiles as in (3.5), then the same argument as in (3.3) shows that $\beta_{i-1}$ and $\beta_{i}$ must agree on the $0^{\text {th }}$ coronas $\mathcal{C}_{0}\left(Q_{i-1}\right)$ and $\mathcal{C}_{0}\left(Q_{i}\right)$ and hence also on the $(k-1)^{s t}$ coronas $\mathcal{C}_{k-1}\left(Q_{i-1}\right)$ and $\mathcal{C}_{k-1}\left(Q_{i}\right)$. Hence, for each $i \geq 1$, the isomorphisms $\beta_{i-1}$ and $\beta_{i}$ agree on $\mathcal{C}_{k-1}\left(Q_{i-1}\right)$ and $\mathcal{C}_{k-1}\left(Q_{i}\right)$.

Now we are almost done. In fact, since $d\left(Q_{i-1}, Q_{i}\right)=1$ for each $i$, a simple induction on $i$ shows that $\beta_{i}$ and $\beta_{0}(=\beta)$ must agree on the $(k-i)^{t h}$ centered corona $\mathcal{C}_{k-i}\left(Q_{i}\right)$, for $i=0,1, \ldots, r$. Hence, since $r \leq k$, the two isomorphisms $\gamma\left(=\beta_{r}\right)$ and $\beta\left(=\beta_{0}\right)$ certainly coincide on $\mathcal{C}_{0}(R)\left(=\mathcal{C}_{0}\left(Q_{r}\right)\right)$. Now it is immediate that $\alpha_{R}:=\left.\gamma\right|_{\mathcal{C}_{0}(R)}=\left.\beta\right|_{\mathcal{C}_{0}(R)}$.

It remains to show that the local mappings of (3.4) fit together coherently to determine a global automorphism of $\mathcal{T}$ which extends $\alpha$. Consider $\varphi: \mathcal{T} \rightarrow \mathcal{T}$ defined by

$$
\left.\varphi\right|_{\mathcal{C}_{0}(Q)}=\alpha_{Q}
$$

where $Q$ is a tile of $\mathcal{T}$ and $\alpha_{Q}$ is as in (3.4).

Lemma 3.7 Let $P, P^{\prime}$ be tiles of $\mathcal{T}$, let $\alpha: \mathcal{C}_{k}(P) \rightarrow \mathcal{C}_{k}\left(P^{\prime}\right)$ be an isomorphism of centered $k^{\text {th }}$ coronas, and let $\varphi: \mathcal{T} \rightarrow \mathcal{T}$ be defined as in (3.6). Then $\varphi$ is a (well-defined) combinatorial automorphism of $\mathcal{T}$. In particular, $\left.\varphi\right|_{\mathcal{C}_{k}(P)}=\alpha$.

Proof: By construction, $\varphi$ assigns to every tile $Q$ of $\mathcal{T}$ a unique tile $Q^{\prime}$, namely $Q^{\prime}:=$ $\varphi(Q)=\alpha_{Q}(Q)$. Moreover, by Lemma 3.6, the restriction of $\varphi$ to $\mathcal{C}_{k}(Q)$ is a (well-defined) isomorphism of centered $k^{\text {th }}$ coronas between $\mathcal{C}_{k}(Q)$ and $\mathcal{C}_{k}\left(Q^{\prime}\right)$, and agrees with the extension $\beta$ of $\alpha$ along a sequence of tiles connecting $P$ to $Q$. In particular, if two tiles $Q$ and $R$ of $\mathcal{T}$ share a facet, then $\alpha_{Q}(F)=\alpha_{R}(F)$ for every face $F$ of this facet.

Now we can prove that $\varphi$ is a well-defined mapping. Let $F$ be any face of $\mathcal{T}$, and let $Q$ and $R$ be arbitrary tiles of $\mathcal{T}$ which contain $F$. By Lemma 2.3 there exists a sequence of tiles

$$
Q=Q_{0}, Q_{1}, \ldots, Q_{n-1}, Q_{n}=Q
$$

each containing $F$, such that $Q_{i-1} \cap Q_{i}$ is a facet of $Q_{i-1}$ and $Q_{i}$ for each $i \geq 1$. Then the above arguments show that $\alpha_{Q_{i-1}}(F)=\alpha_{Q_{i}}(F)$ for each $i \geq 1$. Hence we also have $\alpha_{Q}(F)=\alpha_{R}(F)$. Thus $\varphi$ is well-defined.

The mapping $\varphi$ also preserves incidence of faces. In fact, if $F$ is a face of another face $G$ of a tile $Q$, then $\varphi(F)=\alpha_{Q}(F)$ and $\varphi(G)=\alpha_{Q}(G)$. Hence, since $\alpha_{Q}$ preserves incidence, $\varphi(F)$ must also be a face of $\varphi(G)$. Note that this property holds independent of $Q$.

Moreover, $\varphi$ is invertible. In fact, the mapping $\varphi^{\prime}: \mathcal{T} \rightarrow \mathcal{T}$ induced by $\alpha^{-1}: \mathcal{C}_{k}\left(P^{\prime}\right) \rightarrow$ $\mathcal{C}_{k}(P)$ (in the same way as $\varphi$ by $\alpha$ ) is the inverse of $\varphi$. Note here that $\varphi$ and $\varphi^{\prime}$ each 
preserve adjacency of tiles (along facets), and so does $\varphi^{\prime} \varphi$. Hence, since $\varphi^{\prime} \varphi$ acts like the identity mapping on $\mathcal{C}_{k}(P)$, it must also act like the identity on $\mathcal{C}_{j}(P)$ for each $j>k$ and hence on the whole tiling $\mathcal{T}$. A similar argument applies for $\varphi \varphi^{\prime}$.

It follows that $\varphi$ is a combinatorial automorphism of $\mathcal{T}$. By construction, $\left.\varphi\right|_{\mathcal{C}_{k}(P)}=\alpha$.

The sufficiency proof of Theorem 3.1 is finally complete. In fact, now it is clear that $\mathcal{T}$ is combinatorially tile-transitive. Each (local) isomorphism $\alpha: \mathcal{C}_{k}(P) \rightarrow \mathcal{C}_{k}\left(P^{\prime}\right)$ between the centered $k^{\text {th }}$ coronas of any two tiles $P$ and $P^{\prime}$ extends uniquely to a (global) automorphism of $\mathcal{T}$, namely $\varphi$, which maps $P$ to $P^{\prime}$. When there is no possibility of confusion, we will identify $\varphi$ and $\alpha$ from now on.

It remains to prove the statement of Theorem 3.1 about the stabilizer of a tile $P$ of $\mathcal{T}$. Note here that, if $\alpha \in \Gamma\left(\mathcal{C}_{k}(P)\right.$ ), then our arguments (applied with $P^{\prime}=P$ ) show that $\alpha$ extends uniquely to an automorphism $\varphi$ of $\mathcal{T}$ which fixes $P$; hence $\varphi \in \Gamma_{P}(\mathcal{T})$, or $\alpha \in \Gamma_{P}(\mathcal{T})$ if $\varphi$ is identified with $\alpha$. The other subgroup relationship follows from (2.2). This concludes the proof of Theorem 3.1.

Call a convex $d$-polytope $P$ in $\mathbb{E}^{d}$ combinatorially asymmetric if $\Gamma(P)$ is the trivial group. In this case we must have $d \geq 3$; in fact, $\Gamma(P)$ is the dihedral group of order $2 n$ if $P$ is a convex $n$-gon in the plane. We now have the following corollary.

Corollary 3.8 Let $\mathcal{T}$ be a locally finite face-to-face tiling of $\mathbb{E}^{d}$ by combinatorially asymmetric convex polytopes. Then $\mathcal{T}$ is combinatorially tile-transitive if and only if any two centered $1^{\text {st }}$ coronas of $\mathcal{T}$ are isomorphic (as centered coronas).

Remark 3.9 The coronas of tiles employed in Theorem 3.1 are defined in terms of the distance function $d(.,$.$) of Definition 2.2 for the tiles of \mathcal{T}$. It is worth noting that the statement of Theorem 3.1 remains true (with a similar proof) if the definition of coronas is based instead on the distance function $d_{l}(.,$.$) , for each l \leq d-2$. Altogether this gives $d-2$ variants of the theorem, with the case $l=d-2$ being the original theorem itself. Note that the $k^{\text {th }}$ coronas (with $k$ fixed) get larger as $l$ decreases, so the $k^{\text {th }}$ coronas are smallest when $l=d-2$. However, if $l=d-1$, the analogous theorem fails.

We can already see in the plane why the analogous statement with $l=d-1$ is not true. If $\mathcal{T}$ is a plane tiling by triangles such that at least four triangles meet at each vertex, then the two conditions of Theorem 3.1 for the corresponding coronas (defined by $d_{l}$ with $l=1$ ) are satisfied with $k=1$. On the other hand, there are such tilings $\mathcal{T}$ which are not combinatorially tile-transitive; for example, this occurs if the valencies at the vertices of two distinct tiles do not match up. There are similar examples of tilings by simplices in higher-dimensional spaces.

Remark 3.10 As we mentioned earlier, the convexity of the tiles is not really important. In fact, Theorem 3.1 also extends (with essentially the same proof) to locally finite faceto-face tilings of $\mathbb{E}^{d}$ in which the tiles are topological d-polytopes (homeomorphic images 
of convex d-polytopes). In particular this generalization applies to locally finite face-toface tilings of hyperbolic d-space $\mathbb{H}^{d}$, this being topologically equivalent to $\mathbb{E}^{d}$. Moreover, Theorem 3.1 also holds (with essentially the same proof) for finite face-to-face tilings of spherical d-space $\mathbb{S}^{d}$ by topological d-polytopes; in fact, $\mathbb{S}^{d}$ is simply-connected if $d>1$, and the case $d=1$ is trivial.

\section{The Local Theorem for Tilings}

In the Local Theorem for (face-to-face) Tilings (see Theorem 4.1 below), the tilings $\mathcal{T}$ are monohedral. On the surface, the conditions appearing in our Theorem 3.1 look quite similar to those of Theorem 4.1. Both theorems are local (as their very names indicate), meaning that their conditions involve only certain neighborhoods of tiles but not the whole tiling. However, a closer inspection shows that there are also significant differences. In fact, in Theorem 4.1 the two conditions on the $k^{t h}$ coronas concern global isometries of the ambient space, whereas in Theorem 3.1 they are expressed in terms of local mappings. So, in a sense, the new theorem is "more local" than Theorem 4.1. Moreover, the conditions in Theorem 4.1 only concern the tiles of a corona, whereas in Theorem 3.1 they involve a corona as a whole (that is, as a complex).

Let $\mathcal{T}$ be a locally finite face-to-face tiling, let $P$ be a tile of $\mathcal{T}$, and let $k \geq 0$ be an integer. The $k^{\text {th }}$ tile corona of $P$ in $\mathcal{T}$, denoted by $C_{k}(P)$, is the set of tiles $Q$ of $\mathcal{T}$ with $d(P, Q) \leq k$. Observe here that we are using the term "tile corona" to distinguish the set of tiles $C_{k}(P)$ from the corona $\mathcal{C}_{k}(P)$. The corona $\mathcal{C}_{k}(P)$ is simply the complex of all faces of tiles in the tile corona $C_{k}(P)$. (This differs from [8], where tile coronas are just called coronas.)

As before, the centered $k^{\text {th }}$ tile corona of $P$ is the pair $\left(P, C_{k}(P)\right)$ consisting of $P$, the center of the centered $k^{\text {th }}$ tile corona (usually dropped from the notation), and the $k^{\text {th }}$ tile corona $C_{k}(P)$.

Theorems 3.1 and 4.1 employ concepts of coronas or tile coronas which are defined in terms of the distance function $d(.,$.$) for the tiles of \mathcal{T}$. We mentioned earlier that Theorem 3.1 remains true if, instead of $d(.,$.$) , a distance function d_{l}(.,$.$) with l \leq d-2$ is used to define coronas. The same remark also extends to Theorem 4.1, but now $l=d-1$ is permitted; that is, Theorem 4.1 remains true if the coronas are defined in terms of a distance function $d_{l}(.,$.$) with l \leq d-1$. In the discussion that follows we always take $l=d-2$, that is, the distance function $d(.,$.$) of Definition 2.2.$

Following [8] we say that the centered $k^{\text {th }}$ tile coronas $C_{k}(P)$ and $C_{k}\left(P^{\prime}\right)$ of two tiles $P$ and $P^{\prime}$ of $\mathcal{T}$ are pairwise congruent if there exists a (global) isometry $\alpha$ of the ambient space such that $\alpha(P)=P^{\prime}$ and

$$
C_{k}\left(P^{\prime}\right)=\alpha\left(C_{k}(P)\right)\left(=\left\{\alpha(Q) \mid Q \in C_{k}(P)\right\}\right) .
$$

Let $P$ be a tile of $\mathcal{T}$, and let $G(P)$ be its geometric symmetry group. Let $G_{0}(P):=$ $G(P)$, and for $k \geq 1$ let

$$
G_{k}(P):=\left\{\sigma \in G_{0}(P) \mid \sigma\left(C_{k}(P)\right)=C_{k}(P)\right\} .
$$

THE ELECTRONiC JOURNAL OF COMBINATORICS 11(2) (2004), \#R7 
Then $G_{k}(P)$ consists of those symmetries of $P$ which map each tile in $C_{k}(P)$ to another such tile. It is immediately clear from the definition that we have an infinite chain of subgroups,

$$
G_{P}(\mathcal{T}) \subseteq \ldots \subseteq G_{k}(P) \subseteq G_{k-1}(P) \subseteq \ldots \subseteq G_{1}(P) \subseteq G_{0}(P)=G(P)
$$

with the stabilizer $G_{P}(\mathcal{T})$ of $P$ in the geometric symmetry group $G(\mathcal{T})$ of $\mathcal{T}$ on the left and the (finite) symmetry group $G(P)$ of $P$ on the right.

Recall that a tiling is isohedral if its symmetry group is transitive on the tiles.

Theorem 4.1 A locally finite face-to-face tiling $\mathcal{T}$ of euclidean d-space $\mathbb{E}^{d}$ by convex polytopes is isohedral if and only if there exists a positive integer $k$ with the following properties:

1. Any two centered $k^{\text {th }}$ tile coronas of $\mathcal{T}$ are pairwise congruent.

2. $G_{k}(P)=G_{k-1}(P)$ for each tile $P$ of $\mathcal{T}$.

Moreover, in this case, if $P$ is a tile of $\mathcal{T}$, then $G_{k}(P)=G_{P}(\mathcal{T})$.

The Local Theorem for Tilings is related to the Extension Theorem for Tilings, and both have remarkable consequences and applications. For a detailed discussion see $[1,5$, $6,7,8,9,19]$. The Extension Theorem as well as our Theorem 3.1 rely heavily on the simply-connectedness of the underlying space, but the Local Theorem for Tilings does not (see [6]).

As with Theorem 3.1, convexity of the tiles is not really important in Theorem 4.1 (and, as before, there are also generalizations to tilings in $\mathbb{S}^{d}$ and $\mathbb{H}^{d}$ ). However, if $\mathcal{T}$ actually is a locally finite face-to-face tiling of $\mathbb{E}^{d}$ by convex polytopes, then the integer $k$ in Theorem 4.1 is bounded by a constant $k_{d}$ depending only on $d$ (see [8]); that is, $\mathcal{T}$ is isohedral if and only if the two conditions are met for some $k$ with $k \leq k_{d}$. In fact, if $\mathcal{T}$ is an isohedral face-to-face tiling of $\mathbb{E}^{d}$ by convex polytopes congruent to a $d$-polytope $P$, then the number $f_{d-1}$ of facets of $P$ is bounded (as described below), and hence the order of $G(P)$ is also bounded, each by a constant depending only on $d$. On the other hand, since $G(P)$ is finite, the number of proper ascents in the subgroup chain of (4.1) is bounded by the number $m_{P}$ of prime divisors of $|G(P)|$ (counted with multiplicities), so $k=m_{P}+1$ will always work for $P$. Hence the upper bound for $|G(P)|$ yields an upper bound for $k$. However, in practice, this bound for $k$ is never attained and seems unnecessarily high. In particular, it does not take into consideration the possibility that there are rich point groups which stabilize at an early stage.

If $P$ is a convex $d$-polytope which admits an isohedral face-to-face tiling $\mathcal{T}$ of $\mathbb{E}^{d}$, then

$$
f_{d-1} \leq 2^{d}\left(h-\frac{1}{2}\right)-2,
$$

where $h$ is the index of the translation subgroup in $G(\mathcal{T})$. This bound, due to Tarasov [23], slightly improves the earlier bound of $2^{d}(1+h)-2$ obtained by Delone (see [4]). Since $G(\mathcal{T})$ is among the finitely many crystallographic groups in $\mathbb{E}^{d}$ (up to conjugacy), $h$ 
is uniformly bounded by a constant depending only on $d$. In particular, this yields an upper bound for $f_{d-1}$ depending only on $d$. For example, if $d=3$, the maximum index is 48 and the bound for the number of facets is 378 (Delone's bound yields 390); it is likely that the true bound is considerably lower. The problem of finding space-fillers with the maximum number of facets seems hopelessly difficult. Engel [10] describes some spectacular 3-dimensional space-fillers with 38 facets; however, it is not known if 38 is close to the maximum number possible.

For small dimensions, more is known about the possible values of $k$ in Theorem 4.1. If $\mathcal{T}$ is a tiling of the euclidean plane $\mathbb{E}^{2}$ with polygonal tiles and any two centered $1^{\text {st }}$ tile coronas of $\mathcal{T}$ are pairwise congruent, then $\mathcal{T}$ is isohedral (see [19]); that is, isohedrality is already implied by the first condition of Theorem 4.1 with $k=1$ (the second is not needed). Moreover, the second condition then holds with $k=2$. In euclidean 3 -space $\mathbb{E}^{3}$ there are examples of monohedral tilings by convex polyhedra in which any two centered $1^{\text {st }}$ coronas are pairwise congruent but the tilings are not isohedral (see [11, 12]). For $\mathbb{E}^{3}$ we have the estimate $2 \leq k_{3} \leq 5$ obtained by Shtogrin and Dolbilin (in unpublished work). On the other hand, for the hyperbolic plane $\mathbb{H}^{2}$ there are examples of monohedral tilings by convex pentagons in which any two centered $1^{\text {st }}$ coronas are pairwise congruent but the tilings are not isohedral (see $[2,8]$ ).

It is not known if the number of facets of an arbitrary convex space-filler of $\mathbb{E}^{d}$ (with $d \geq 3$ ) is bounded by a constant depending only on $d$. In theory, this leaves the possibility that there are space-fillers with arbitrarily large symmetry groups. Clearly, a face-to-face tiling $\mathcal{T}$ by congruent copies of such a space-filler $P$ cannot be isohedral. In particular, for such tilings $\mathcal{T}$, no integer $k$ will satisfy both properties of Theorem 4.1 simultaneously. In theory, then, there may exist sequences of tilings $\left(\mathcal{T}_{j}\right)_{j \geq 1}$ and corresponding space-fillers $\left(P_{j}\right)_{j \geq 1}$, as well as an increasing sequence of integers $\left(k_{j}\right)_{j \geq 1}$, such that the first condition of Theorem 4.1 holds with $k=k_{j}$ while the second holds not for $k_{j}$ but only for an integer larger than $k_{j}$. Therefore, at present we cannot determine if there also exists an upper bound $k_{d}^{\prime}$ for the integers $k$ with the property that a tiling with pairwise congruent centered $k^{\text {th }}$ tile coronas is necessarily isohedral.

It is an interesting question to what extent the above remains true for our Theorem 3.1. Now, if $l_{P}$ denotes the number of prime divisors of $|\Gamma(P)|$ (counted with multiplicities), then the number of proper ascents in the subgroup chain of $(2.2)$ is bounded by $l_{P}$, and hence we can always take $k=l_{P}+1$ in Theorem 3.1. However, for a combinatorially tiletransitive face-to-face tiling of $\mathbb{E}^{d}$ by convex polytopes, the order of the automorphism group $\Gamma(P)$ of a tile $P$ need not be bounded by a constant depending only on $d$. In fact, for each $p \geq 3$, the prism $P$ over a convex $p$-gon (with group order $|\Gamma(P)|=4 p$ ) admits a combinatorially tile-transitive tiling $\mathcal{T}$ of $\mathbb{E}^{3}$ obtained from layers of plane tilings by convex $p$-gons, where a fixed number $q$ of $p$-gons meet at each vertex, provided $\frac{1}{p}+\frac{1}{q} \leq \frac{1}{2}$; these plane tilings are isomorphic copies of the regular tessellations $\{p, q\}$ of the euclidean or hyperbolic plane (see $[16,21]$ ). However, if $p>6$, such a tiling $\mathcal{T}$ constructed from layers of $\{p, q\}$ must necessarily be non-normal; recall that a tiling is normal if its tiles are uniformly bounded, meaning that there are positive parameters $r, R$ such that each tile contains a ball of radius $r$ and is contained in a ball of radius $R$ (see [15]). 
In this context we mention the following interesting open problem. Suppose a convex $d$-polytope $P$, with $d>2$, admits a combinatorially tile-transitive face-to-face tiling of $\mathbb{E}^{d}$ which is normal. Is it true, then, that the order of the automorphism group $\Gamma(P)$ is bounded by a constant depending only on $d$ ?

For $p \geq 3$, normal monotypic face-to-face tilings of $\mathbb{E}^{3}$ by prisms $P$ over convex $p$-gons can be obtained by the projection technique described in [20, Thm.3]. These tilings $\mathcal{T}$ are not combinatorially tile-transitive; however, $G(\mathcal{T})$ contains an infinite discrete reflection group as a subgroup, so $\mathcal{T}$ has only finitely many transitivity classes of tiles under $G(\mathcal{T})$ and hence $\Gamma(\mathcal{T})$. The tilings are produced by projection from the boundary complex of a convex 4-polytope with prisms as facets, namely the rectangular product of a pair of regular $p$-gons contained in orthogonal 2-planes in $\mathbb{E}^{4}$.

Acknowledgments: The research was done, in part, while the authors attended a research program on "The Mathematics and Physics of Long-Range Aperiodic Order" at the University of Greifswald and the Krupp-Kolleg in Greifswald. The authors would like to thank these institutions for their hospitality. Our gratitude also extends to the program organizers: Michael Baake, Uwe Grimm, Robert V. Moody, Alexander Quandt, and Janusz Wolny.

We also thank two anonymous referees for their helpful comments.

\section{References}

[1] A.D.Alexandrov, On filling of space by polytopes (in Russian). Zapisky Leningradskogo Universiteta (Notes of Leningrad University) Ser. Math. Phys. Chem. 9 (1954), $33-43$.

[2] K.Böröczky, Gömbkitöltések állandó görbületü terekben, I,II. Mat. Lapok 25 (1974), 265-306, and 26 (1974), 67-90.

[3] L.Danzer, B.Grünbaum and G.C.Shephard, Does every type of polyhedron tile threespace. Structural Topology 8 (1983), 3-14.

[4] B.N.Delone and N.N.Sandakova, Theory of stereohedra. Proc. Steklov Inst. 64 (1961), 28-51 (in Russian).

[5] B.N.Delone, N.P.Dolbilin, M.I.Shtogrin and R.V.Galiulin, A local criterion for regularity of a system of points. Soviet Math. Dokl. 17 (1976), 319-322.

[6] N.Dolbilin, The Extension Theorem. Discrete Mathematics 221 (2000), 43-59.

[7] N.P.Dolbilin and V.S.Makarov, The extension theorem in the theory of regular tilings and its applications. Proceedings of Steklov Math. Inst. 239 (2002), 136-158.

[8] N.Dolbilin and D.Schattschneider, The Local Theorem for Tilings. In Quasicrystals and Discrete Geometry (J.Patera, ed.), Fields Institute Monographs, Vol. 10, Amer. Math. Soc. (1998), 193-199. 
[9] N.P.Dolbilin, J.C.Lagarias and M.Senechal, Multiregular point systems. Discrete Comput. Geom. 20 (1998), 477-498.

[10] P.Engel, Über Wirkungsbereiche von kubischer Symmetrie. Zeitschrift für Kristallographie 154 (1981), 199-215.

[11] P.Engel, Geometric Crystallography. Reidel Publishing Company (Dordrecht, 1986).

[12] P.Engel, Mathematical problems in modern crystallography. Crystal symmetries. Comput. Math. Appl. 16 (1988), no. 5-8, 425-436.

[13] B.Grünbaum and G.C.Shephard, Incidence symbols and their applications. Proc. Symp. Pure Math. 34 (1979), 199-244.

[14] B.Grünbaum and G.C.Shephard, Tilings with congruent tiles. Bull. Amer. Math. Soc. N.S. 3 (1980), 951-973.

[15] B.Grünbaum and G.C.Shephard, Tilings and Patterns. Freeman \& Co. (New York, 1986).

[16] B.Grünbaum, P.Mani-Levitska and G.C.Shephard, Tiling three-dimensional space with polyhedral tiles of a given isomorphism type. J. London Math. Soc. (2) 29 (1984), 181-191.

[17] P. McMullen and E. Schulte, Abstract Regular Polytopes. Encyclopedia of Mathematics and its Applications, 92, Cambridge University Press (Cambridge, 2002).

[18] M.A.Perles and G.C.Shephard, Facets and nonfacets of convex polytopes. Acta Mathematica 119 (1967), 113-145.

[19] D.Schattschneider and N.Dolbilin, One corona is enough for the euclidean plane. In Quasicrystals and Discrete Geometry (J.Patera, ed.), Fields Institute Monographs, Vol. 10, Amer. Math. Soc. (1998), 207-246.

[20] E.Schulte, Nontiles and nonfacets for euclidean space, spherical complexes and convex polytopes. J. Reine Angewandte Mathematik 352 (1984), 161-183.

[21] E.Schulte, Tiling three-space by combinatorially equivalent convex polytopes. Proc. London Math. Soc. (3) 49 (1984), 128-140.

[22] R.P.Stanley, Enumerative Combinatorics, Vol. I, Wadsworth \& Brooks/Cole, (Monterey, 1986).

[23] A.S.Tarasov, Complexity of convex stereohedra. Mathematical Notes 61 (5), 668-671.

[24] G.Ziegler, Lectures on Polytopes. Springer-Verlag (New York, 1994). 